\title{
The Space Weather \& Ultraviolet Solar Variability Microsatellite Mission (SWUSV)
}

Luc Damé ${ }^{1}$ and The SWUSV Team (Mustapha Meftah, Alain Hauchecorne, Philippe Keckhut, Alain Sarkissian, Marion Marchand, Abdenour Irbah, Éric Quémerais, Slimane Bekki, Thomas Foujols, Matthieu Kretzschmar, Gaël Cessateur, Alexander Shapiro, Werner Schmutz, Sergey Kuzin, Vladimir Slemzin, Sergey Bogachev, José Merayo, Peter Brauer, Kanaris Tsinganos, Antonis Paschalis, Ayman Mahrous, Safinaz A. Khaled, Ahmed Ghitas, Besheir Marzouk, Amal Zaki, Ahmed A. Hady, Rangaiah Kariyappa)

${ }^{1}$ LATMOS/IPSL/CNRS/UVSQ, 11 Boulevard d'Alembert, 78280 Guyancourt, France email: luc.dame@latmos.ipsl.fr

\begin{abstract}
We present a summary of the scientific objectives, payload and mission profile of the Space Weather \& Ultraviolet Solar Variability Microsatellite Mission (SWUSV) proposed to CNES and ESA (small mission).
\end{abstract}

Keywords. Space Weather, solar flares, Coronal Mass Ejections, solar variability, ultraviolet.

\section{Objectives}

The SWUSV mission encompasses three major scientific objectives: (1) Space Weather including the prediction and detection of major eruptions and coronal mass ejections (Lyman-Alpha and Herzberg continuum imaging); (2) solar forcing on the climate through radiation and their interactions with the local stratosphere (UV spectral irradiance from 180 to $400 \mathrm{~nm}$ by bands of $20 \mathrm{~nm}$, plus Lyman-Alpha and the CN bandhead); (3) simultaneous radiative budget of the Earth, UV to IR, with an accuracy better than $1 \%$ in differential.

SWUSV is targeting the observation of the Space environment, and in particular the premisses, the very early start ("precursor indicators"), of the coronal mass ejections, the interplanetary ones (ICMEs), that are the most important since the only ones with a destructive impact potential on Earth. With Lyman-Alpha (and H-Alpha on ground in complement, 2 major solar lines formed at different heights in the solar chromosphere), we should be able to better predict, hours in advance, the major flares and ICMES and their geoeffectiveness.

Concerning the solar influence on climate, the ultraviolet part of the spectra $(<350 \mathrm{~nm})$ is the only one having its energy completely absorbed in the high atmosphere (stratosphere), in the ozone layer and in the oxygen bands (Herzberg continuum at 200-220 $\mathrm{nm})$. The large variability of UV wavelengths over the solar cycle $(5-10 \%$ at $200-220$ $\mathrm{nm}$, factor 2 at Lyman-Alpha) is probably at the origin of a solar influence on climate, the UV affecting the temperatures and the stratospheric dynamic (the response to the solar heating extends to the poles and down, to the tropopause, modifying planetary waves and meteorological conditions). With these UV observations, and when coupled to a complete local radiative budget from UV to IR, we shall be able to characterize this solar influence to its full dynamical extent. 


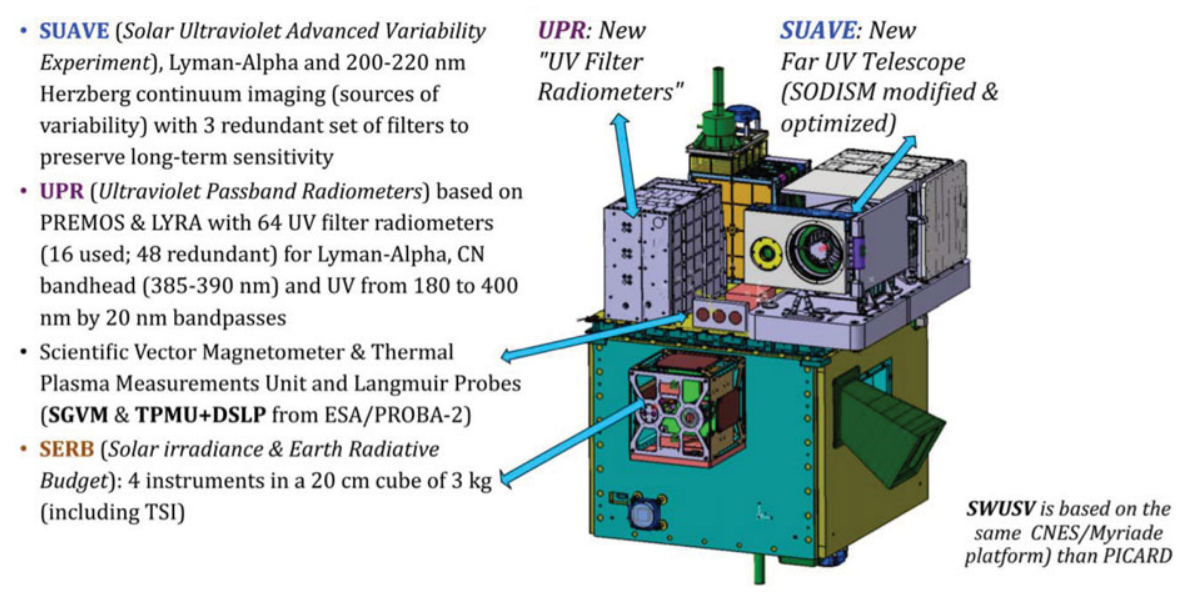

Figure 1. Illustration of the possible accommodation of the SWUSV instruments on the CNES/Myriade microsatellite platform (dimensions $600 \times 600 \times 800 \mathrm{~mm}^{3}$ ).

\section{Model Payload \& Mission Profile}

SWUSV includes 5 instruments: SUAVE (Solar Ultraviolet Advanced Variability Experiment), an optimized telescope for FUV (Lyman-Alpha) and MUV (200-220 nm Herzberg continuum) imaging (sources of variability); UPR (Ultraviolet Passband Radiometers), with 64 UV filter radiometers; a vector magnetometer; thermal plasma measurements and Langmuir probes; and a total and spectral solar irradiance and Earth radiative budget ensemble (SERB, Solar irradiance \& Earth Radiative Budget). The model payload and microsatellite (CNES/Myriade platform on a Sun synchronous orbit comparable to PICARD: altitude, $725 \mathrm{~km}$; inclination, $98.29^{\circ}$; local time of ascending node, $6 \mathrm{~h} 00+/-$ 10 minutes) are presented in Fig. 1.

\section{Conclusion}

The microsatellite investigation SWUSV is responding to the need to understand the influence of stratospheric dynamics on the climate by providing the tools to measure and quantify the influence of UV variability and determine its origin. It also carries, through Lyman-Alpha imaging, probably the best indicator for precursor signs of major Space Weather events. The program has the advantage of relying on technological developments made in very recent microsatellite missions of CNES (PICARD) and ESA (PROBA-2), and on a long expertise of the proposing team in far UV imaging (Lyman-Alpha in particular but also Herzberg continuum at $220 \mathrm{~nm}$ ). The SWUSV mission is presented in detail in Damé and The SWUSV Team (2013).

\section{Acknowledgements}

We are grateful to the CNES that supports the SWUSV initiative with a R\&D program on far ultraviolet solar telescopes design and performances.

\section{Reference}

Damé, L., The SWUSV Team (Meftah, M., Hauchecorne, A., Keckhut, P., Sarkissian, A., Marchand, M., Irbah, A., Quémerais, E., Bekki, S., Foujols, T., Kretzschmar, M., Cessateur, G., Shapiro, A., Schmutz, W., Kuzin, S., Slemzin, V., Urnov, A., Bogachev, S., Merayo, J., Brauer, P., Tsinganos, K., Paschalis, A., Mahrous, A., Khaled, S. A., Ghitas, A., Marzouk, B., Zaki, A., Hady, A. A., \& Kariyappa, R.) 2013, Journal of Adv. Research, 4, 235 